
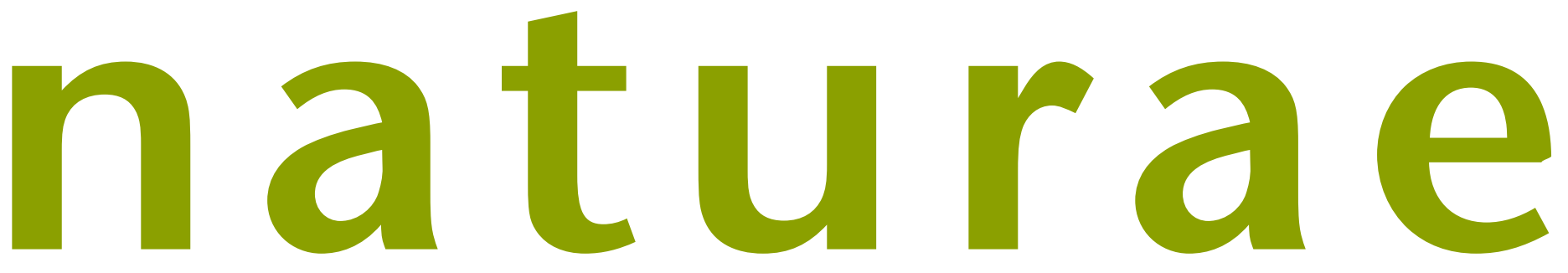

$2021 \cdot 23$

Hether

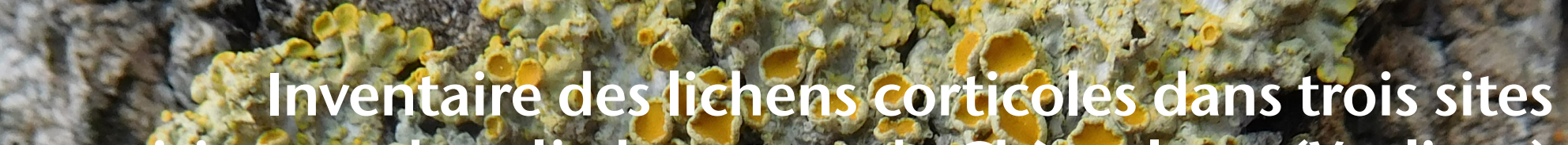
i par sienseet dons la bonetum de ghéveloup (Vvelines) 2) f.jS Elise LEBRELON, Simon RIVART, Sébastien LEBLOND \& Caroline MEYER

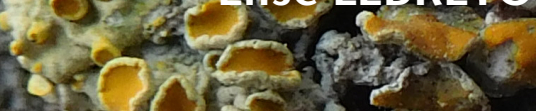

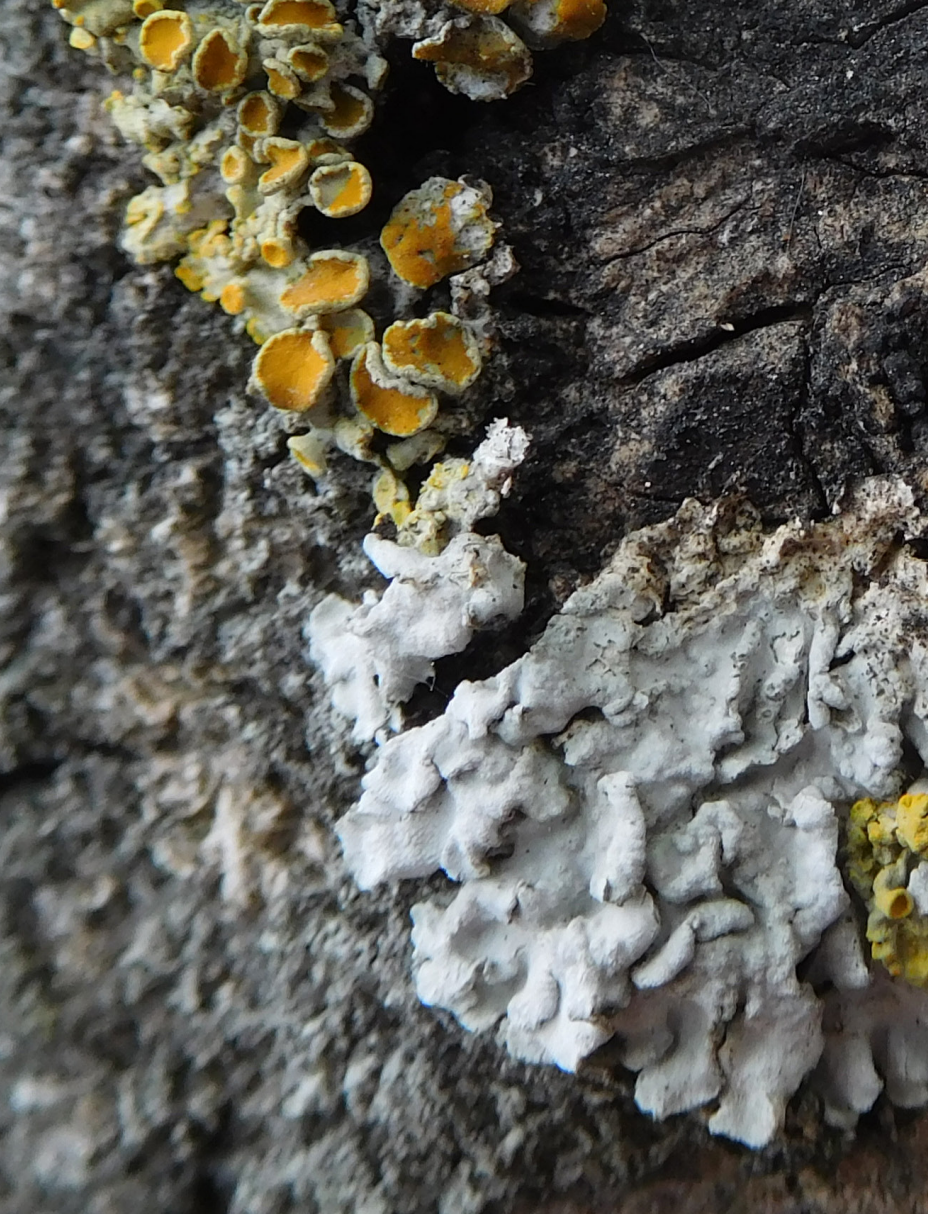

art. 2021 (23) - Publié le 3 novembre 2021 www. revue-naturae.fr
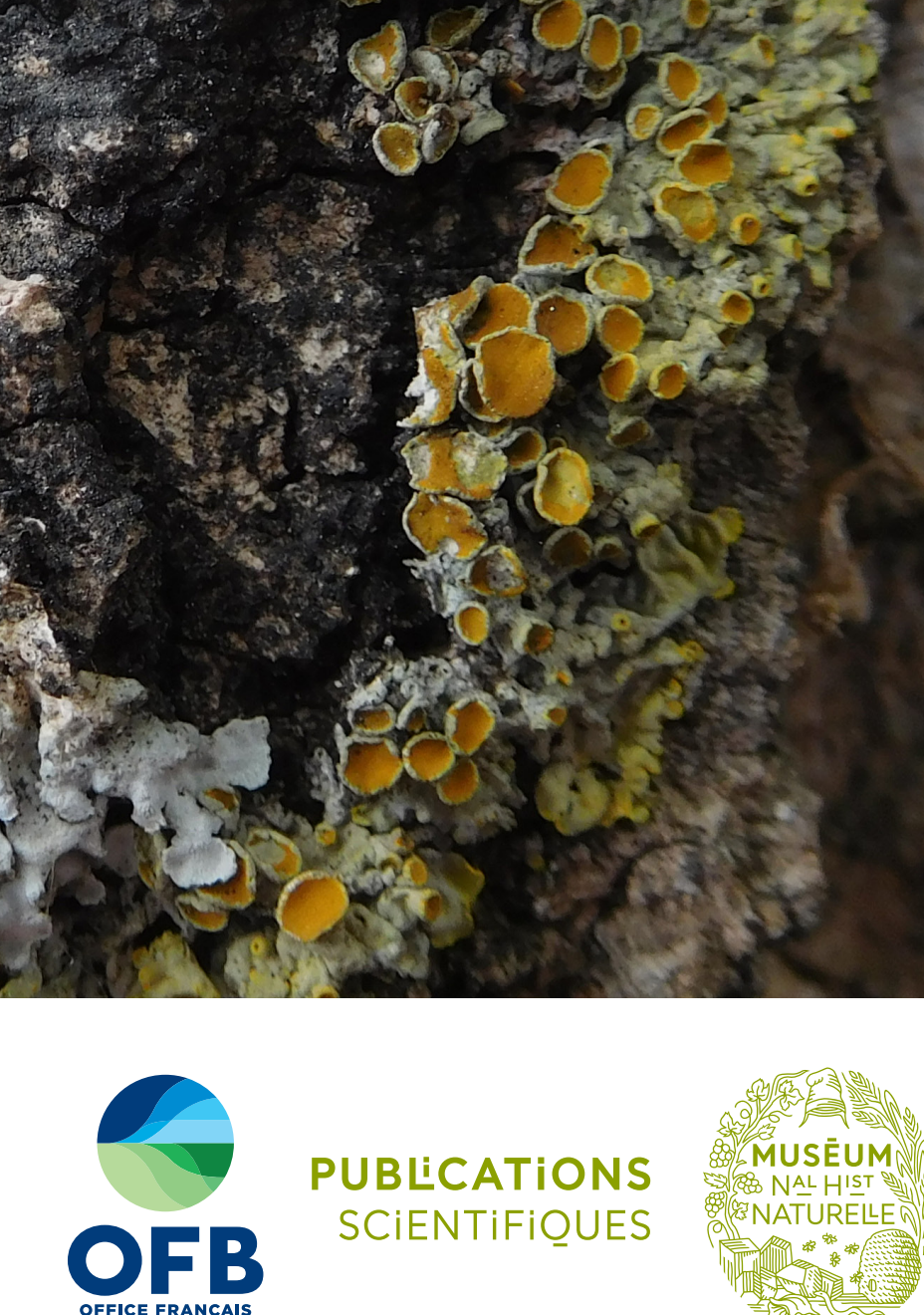
DiRECTEUR DE LA PUBLICATION / PUBLICATION DIRECTOR: Bruno David,

Président du Muséum national d'Histoire naturelle

RÉDACTEUR EN CHEF / EDITOR-IN-CHIEF: Jean-Philippe Siblet

ASSISTANTE DE RÉDACTION / ASSISTANT EDITOR: Sarah Figuet (naturae@mnhn.fr)

Mise EN PAGE / PAGE LAYOUT: Sarah Figuet

COMITÉ SCIENTIFIQUE / SCIENTIFIC BOARD:

Luc Abbadie (UPMC, Paris)

Luc Barbier (Parc naturel régional des caps et marais d'Opale, Colembert)

Aurélien Besnard (CEFE, Montpellier)

Vincent Boullet (Expert indépendant flore/végétation, Frugières-le-Pin)

Hervé Brustel (École d'ingénieurs de Purpan, Toulouse)

Patrick De Wever (MNHN, Paris)

Thierry Dutoit (UMR CNRS IMBE, Avignon)

Éric Feunteun (MNHN, Dinard)

Romain Garrouste (MNHN, Paris)

Grégoire Gautier (DRAAF Occitanie, Toulouse)

Olivier Gilg (Réserves naturelles de France, Dijon)

Frédéric Gosselin (Irstea, Nogent-sur-Vernisson)

Patrick Haffner (PatriNat, Paris)

Frédéric Hendoux (MNHN, Paris)

Xavier Houard (OPIE, Guyancourt)

Isabelle Leviol (MNHN, Concarneau)

Francis Meunier (Conservatoire d'espaces naturels - Hauts-de-France, Amiens)

Serge Muller (MNHN, Paris)

Francis Olivereau (DREAL Centre, Orléans)

Laurent Poncet (PatriNat, Paris)

Nicolas Poulet (OFB, Vincennes)

Jean-Philippe Siblet (PatriNat, Paris)

Laurent Tillon (ONF, Paris)

Julien Touroult (PatriNat, Paris)

COUVERTURE / COVER:

Xanthoria parietina (L.) Th.Fr. et Physconia grisea (Lam.) Poelt sur Peuplier noir, Populus nigra var. italica Du Roi en bord de Seine (Paris).

Crédit photo: E. Lebreton.

Naturae est une revue en flux continu publiée par les Publications scientifiques du Muséum, Paris

Naturae is a fast track journal published by the Museum Science Press, Paris

Les Publications scientifiques du Muséum publient aussi / The Museum Science Press also publish:

Adansonia, Zoosystema, Anthropozoologica, European Journal of Taxonomy, Geodiversitas, Cryptogamie sous-sections Algologie, Bryologie, Mycologie, Comptes Rendus Palevol.

Diffusion - Publications scientifiques Muséum national d'Histoire naturelle

CP 41 - 57 rue Cuvier F-75231 Paris cedex 05 (France)

Tél. : 33 (0)1 40794805 / Fax: 33 (0)1 40793840

diff.pub@mnhn.fr / https://sciencepress.mnhn.fr

(c) Publications scientifiques du Muséum national d'Histoire naturelle, Paris, 2021

ISSN (électronique / electronic): 1638-9387 


\title{
Inventaire des lichens corticoles dans trois sites parisiens et dans l'arboretum de Chèvreloup (Yvelines)
}

\author{
Élise LEBRETON \\ Simon RIVART \\ Sébastien LEBLOND \\ Caroline MEYER \\ PatriNat, Office français de la Biodiversité, CNRS, Muséum national d'Histoire naturelle, \\ 12 rue Buffon, F-75005 Paris cedex 05 (France) \\ eliselebreton@yahoo.fr \\ simon.rivart@gmail.com \\ sebastien.leblond@mnhn.fr \\ caroline.meyer@mnhn.fr
}

Soumis le 11 octobre 2020 | Accepté le 8 février 2021 | Publié le 3 novembre 2021

MOTS CLÉS

Lichens,

milieu urbain, poussières,

Paris,

Yvelines.

KEY WORDS

Lichens,

urban environment

dust,

Yvelines.
Lebreton E., Rivart S., Leblond S. \& Meyer C. 2021. - Inventaire des lichens corticoles dans trois sites parisiens et dans l'arboretum de Chèvreloup (Yvelines). Naturae 2021 (23): 321-332. https://doi.org/10.5852/naturae2021a23

\section{RÉSUMÉ}

Cet article présente le résultat des inventaires des lichens corticoles réalisés dans le cadre du programme LiPaChE (évolution spatio-temporelle de la diversité Lichénique Parisienne en lien avec les Changements Environnementaux). Des arbres d'alignement, appartenant à huit essences d'arbres communes des parcs, ont été examinés dans trois sites parisiens (jardin des Plantes, jardin du Luxembourg et bords de Seine) et à l'arboretum de Chèvreloup dans les Yvelines. La diversité lichénique a été observée sur les troncs avec deux protocoles: IAP (Index of Atmospheric Purity) et AFNOR (Association française de Normalisation). Les résultats de cette étude concernent 61 espèces de lichens (43 espèces à Paris et 43 espèces à Chèvreloup), dont cinq constituent des premières mentions départementales. La présence de lichens saxicoles, à la base de certains troncs, ainsi que l'effet des paramètres environnementaux sur le cortège d'espèces retrouvées, sont discutés.

\section{ABSTRACT}

Inventory of corticolous lichens in three Parisian sites and in the arboretum of Chèreloup (Yvelines).

This article presents the results of the inventories that were carried out within the programme LiPaChE (Lichens du bassin Parisien Changement Environnement). Three Parisian stations, (jardin des Plantes, jardin du Luxembourg and the banks of the Seine river) and the Chèvreloup arboretum in the Yvelines were visited. These inventories were conducted on tree avenues, belonging to eight tree species common to the parks. The trees were sampled around the trunk, from the base to $2 \mathrm{~m}$ high with two protocols. The results of this study concern 61 lichens species ( 43 species in Paris and 43 species in the Yvelines station). Three species are new for Paris: Flavoparmelia soredians (Nyl.) Hale, Lecanora compallens van Herk \& Aptroot and Rinodina gennarii Bagl., and five species constitute first departmental records: Caloplaca arnoldii (Wedd.) Zahlbr. ex Ginzb., Caloplaca flavocitrina (Nyl.) H. Olivier, Endocarpon adscendens (Anzi) Müll. Arg., Lecanora leptyrodes (Nyl.) Degel. and Xanthomendoza huculica (S.Y.Kondr) Diederich. A rare species, Toninia populorum (A. Massal.) Kistenich, Timdal, Bendiksby \& S. Ekman and common species Caloplaca cerinella (Nyl.) Flagey, Myriolecis crenulata (Hook.) Śliwa, Zhao Xin \& Lumbsch were reported a century after they were last mentioned in Paris. The influence of environmental parameters on lichens is discussed, in particular the presence of saxicolous lichens on trees in dusty areas of parks. 


\section{INTRODUCTION}

Les lichens sont des organismes symbiotiques: une algue ou une cyanobactérie (ou les deux exceptionnellement) et un champignon vivent dans une association produisant une structure originale appelée "thalle» (McMullin \& Anderson 2014). Ces organismes sont ubiquistes, ils colonisent tous les écosystèmes terrestres, depuis les bioclimats polaires en Arctique (Daniels et al. 2000; Kristinsson et al. 2010) et en Antarctique (Bokhorst et al. 2016), jusqu'aux bioclimats tropicaux (Cáceres et al. 2017), en passant par les déserts (Ryan et al. 2004). Ils se développent sur différents substrats naturels: roches (lichens saxicoles), écorces (lichens corticoles), bois (lichens lignicoles), sol (lichens terricoles ou detriticoles), feuilles (lichens foliicoles), ou d'origine anthropique: mortiers, toitures, plastiques, verre, etc. Au niveau mondial, le nombre d'espèces de lichens acceptés selon la classification des champignons lichénisés de 2016 est de 19387 répartis dans 995 genres, 115 familles, 39 ordres et huit classes (Lücking et al. 2016) ; cependant, la diversité lichénique est peu étudiée et cette évaluation est probablement très inférieure aux valeurs réelles (Sipman $\&$ Aptroot 2001; Lücking et al. 2016).

Les lichens n'ont ni racine ni cuticule: ils sont dépendants des éléments nutritifs provenant de l'eau de pluie, de l'air ou parfois de leur support (Richardson 1975; Sloof 1993; Richardson \& Cameron 2004). Ne possédant pas de stomates, ils ne régulent pas leurs échanges gazeux avec l'atmosphère et sont exposés à toutes les substances présentes dans l'air ambiant. De ce fait, ils sont très fortement dépendants des conditions environnementales (Purvis 2000) et sont particulièrement sensibles aux facteurs abiotiques liés aux conditions macro et microclimatiques s'exerçant dans le milieu (Pinho et al. 2004; Giordani 2007). C'est pourquoi, depuis les observations de Nylander (1866), ils sont utilisés comme organismes sentinelles de la qualité de l'air.

En Île-de-France, la majorité des inventaires portant sur la lichénofonge a été réalisée entre le XVII ${ }^{\mathrm{e}}$ siècle et les années 1990. Il n'existe de fait que peu de données sur la biodiversité actuelle. Tournefort en 1698 mentionne une trentaine d'espèces de lichens en Île-de-France (Tournefort 1698). Un siècle plus tard, Mérat et Chevallier publient d'importants recueils sur l'île-de-France avec un total de 349 espèces de lichens (Mérat 1812; Chevallier 1836). Néanmoins, leurs identifications seront remises en cause par Nylander (1896) en raison de nombreuses indications erronées et d'identifications inexactes dues à des déterminations basées sur des caractères macroscopiques et non microscopiques. En 1896, Nylander constate une disparition des lichens corticoles jadis observés au jardin du Luxembourg, qu'il attribue aux récentes constructions autour du jardin et au "noir de fumée» émanant des usines avoisinantes (Nylander 1896). D’autres auteurs tels que Doignon et Bouly de Lesdain, poursuivent les inventaires dans et autour de Paris jusqu'au milieu du $\mathrm{xx}^{\mathrm{e}}$ siècle (Doignon 1947 ; Bouly de Lesdain 1948). La plupart des échantillons récoltés à Paris sont conservés dans l'herbier du Muséum national d'Histoire naturelle de Paris (herbier PC). À partir des années 1970, les études ont eu pour objet la biosurveillance de la qualité de l'air et se sont basées sur la notation de la présence ou de l'absence d'espèces corticoles indicatrices définies au sein de listes plus ou moins précises sur le plan taxonomique (Bélair 1977; Déruelle 1983; PartiCitaE 2019). Ces dernières études constituent donc un matériel intéressant sur le plan de la bioindication de la distribution de certains taxons, mais ne permettent pas de documenter de manière large la diversité spécifique actuelle des lichens en Île-de-France.

Dans le cadre du programme LiPaChE initié en 2017 (évolution spatio-temporelle de la diversité Lichénique parisienne en lien avec les Changements Environnementaux) soutenu par l' Institut d'Aménagement et d'Urbanisme de la région Île-de-France (IAU IdF) et l'Action transversale du Muséum (ATM), nous avons étudié l'évolution spatio-temporelle de la diversité spécifique de la lichénofonge corticole à Paris en relation avec les pressions environnementales (changement climatique, pollution atmosphérique, urbanisme) depuis 150 ans. Dans cet article nous présenterons plus particulièrement les résultats des inventaires effectués en 2018 à Paris et à l'arboretum de Chèvreloup (Le ChesnayRocquencourt, 78150), dans le cadre d'un stage de master (Lebreton et al. 2018). Ces inventaires se sont concentrés sur des arbres d'alignement, appartenant à huit essences d'arbres communes des parcs.

\section{MATÉRIEL ET MÉTHODES}

\section{SITES D'ÉTUDES}

Trois sites parisiens et un site dans les Yvelines ont été étudiés: le jardin des Plantes (JDP), le jardin du Luxembourg (JDL), les bords de Seine (BDS) et l'arboretum de Chèvreloup (AC) (Fig. 1).

Les trois sites parisiens sont historiquement bien documentés: le JDP et le JDL ont été prospectés au XIXe siècle (Nylander 1896), les BDS en 1946 (Bouly de Lesdain 1948). L'arboretum de Chèvreloup se situe à côté du jardin de Versailles, lieu qui fut inventorié à plusieurs reprises (Bouly de Lesdain 1905, 1907, 1908, 1912, 1921). Les sites ont été sélectionnés selon deux critères: présence de cinq arbres d'alignement de la même espèce et présence d'au moins une espèce commune entre les sites. Dans deux sites, seulement trois ou quatre arbres d'alignement ont pu être prospectés.

La nature de la couche superficielle du sol - ou revêtement - qui entoure les arbres, varie d'un site à l'autre et à l'intérieur d'un même site d'étude. Parmi ces quatre sites, seuls les bords de Seine ne sont pas interdits aux chiens. Les arbres présentent donc une "zone canine», comprise entre 0 et $10 \mathrm{~cm}$ sur laquelle les chiens peuvent uriner enrichissant ainsi l'écorce en urée. La fréquentation canine, uniquement relevée dans ce site, limite l'installation et le développement des lichens sur la base des arbres (Fredon et al. 1993; Ishiomin \& Letrouit-Galinou 1997).

Dans le cadre de cet inventaire lichénique, 20 arbres d'alignement ont été prospectés au JDL, 13 au JDP, 20 sur les bords de Seine et 30 à Chèvreloup. 


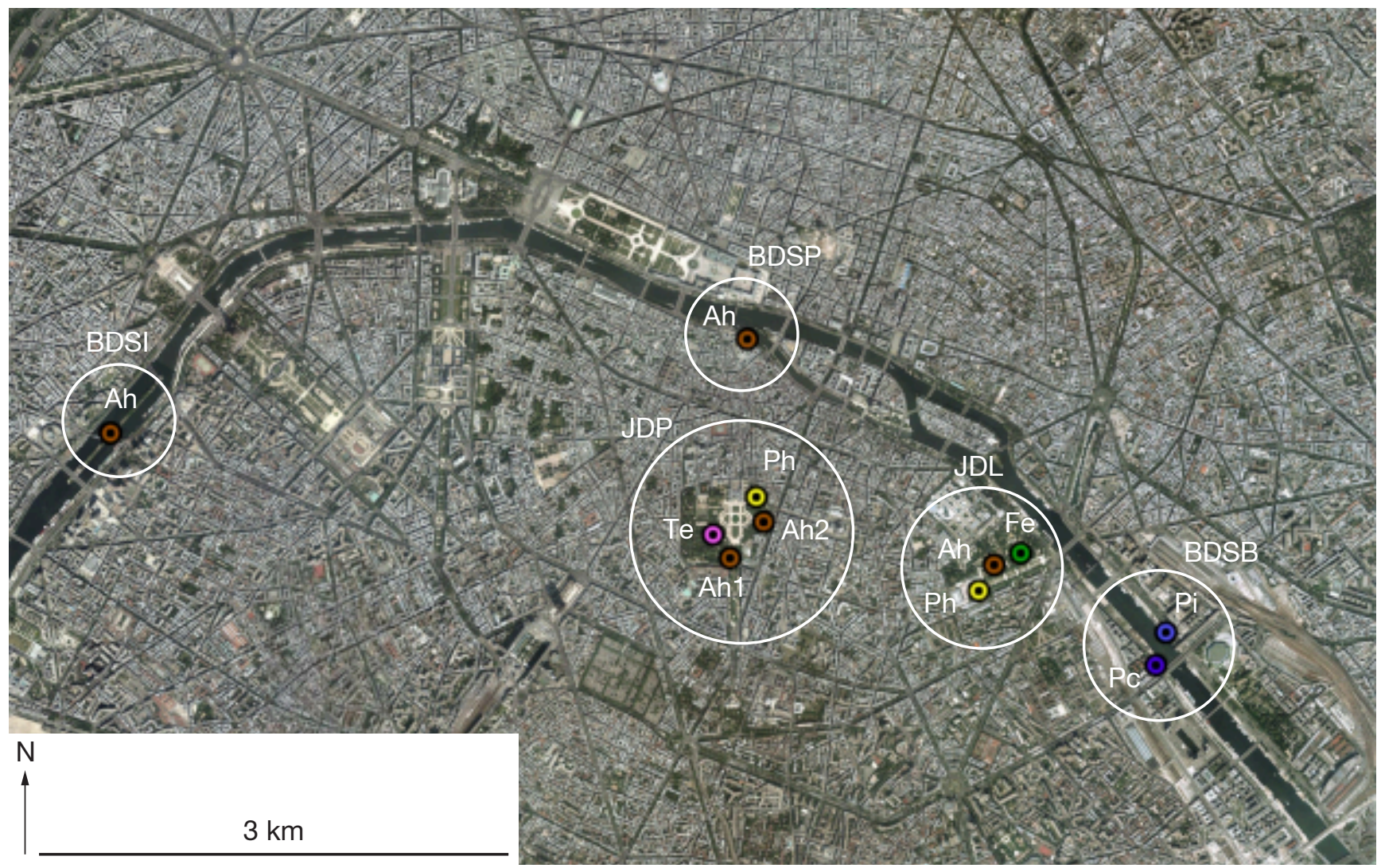

FIG. 1. - Localisation des sites d'études parisiens et des essences d'arbres prospectées. Abréviations: essences d'arbres: Ah, Aesculus hippocastanum L. (Marronier d'Inde); Fe, Fraxinus excelsior L. (Frêne commun); Pc, Populus $\times$ canescens (Aiton) Sm. (Peuplier grisard); Ph, Platanus $\times$ hispanica Mill. ex Münchh. (Platane); Pi, Populus nigra var. italica Du Roi (Peuplier noir); Te, Tilia $\times$ europaea L. (Tilleul); stations: BDSB, bords de Seine station Bercy; BDSI, bords de Seine station Île aux Cygnes; BDSP, bord de Seine station Pont-Neuf; JDL, jardin du Luxembourg; JDP, jardin des Plantes. Chaque point correspond à cinq arbres, sauf Te qui correspond à trois arbres. Vue satellitaire: GoogleEarth.

\section{Les bords de Seine}

La Seine coupe Paris en son milieu sur $13 \mathrm{~km}$ et sa largeur varie de 30 à $200 \mathrm{~m}$. Le site des bords de Seine a été divisé en trois stations, Bercy, Pont-Neuf et Île aux Cygnes, distantes les unes des autres d'environ 3-4 km (Fig. 1).

La station Bercy et la station Pont-Neuf éloignées de $3 \mathrm{~km}$ sont similaires. Les arbres prospectés sur ces deux stations sont: cinq marronniers (Aesculus hippocastanum L.) et dix peupliers (cinq Populus nigra var. italica Du Roi et cinq Populus $\times$ canescens (Aiton) Sm.). Ces arbres sont localisés sur les berges bitumées, soumises aux crues de la Seine. La route est au-dessus de la berge, sur le quai haut. Les arbres sont placés à $1 \mathrm{~m}$ d'un mur et à $3 \mathrm{~m}$ de la Seine.

L'Île aux Cygnes est située au milieu de la Seine et n'est pas impactée par les crues. Cette station se présente sous la forme d'un chemin pavé qui est encadré par deux grandes allées d'arbres plantés en terre. Cinq marronniers y ont été prospectés.

\section{Le jardin des Plantes}

Le jardin des Plantes, ouvert au public, est situé dans le 5e arrondissement de Paris. Dans les deux grandes allées de platanes (Platanus $\times$ hispanica Mill. ex Münchh.), cinq individus ont été prospectés ainsi que cinq marronniers dans une autre allée. Les trois Frênes communs (Fraxinus excelsior L.) situés dans l'allée des marronniers ont été observés (Fig. 1). Avec plus de 3 millions de visiteurs par an (Goldhaber \& Ramdane 2018), le jardin des Plantes est un lieu très fréquenté. Par conséquent, les arbres sont taillés chaque année pour éviter les chutes de branches et le sol comporte un revêtement calcaire pulvérulent et volatile qui contribue, par son piétinement, à un empoussièrement de la base des troncs.

\section{Le jardin du Luxembourg}

Le jardin du Luxembourg, ouvert au public, est situé dans le $6^{e}$ arrondissement de Paris. Les inventaires ont été réalisés dans quatre parties du jardin caractérisées par l'essence du phorophyte et le revêtement du sol. La première partie du jardin concerne l'allée Gay-Lussac, où les cinq marronniers prospectés sont plantés dans un sol avec un revêtement calcaire pulvérulent, fortement émetteur de poussières. Les cinq autres marronniers ont été prospectés dans la seconde partie du jardin, l'allée de l'observatoire où les arbres sont plantés sur un mélange d'argile et de calcaire. La présence de larges bandes de pelouse à proximité directe des arbres est observée. Les cinq tilleuls étudiés, dans la troisième partie du jardin située vers le terrain de tennis et le poste de garde, ont 


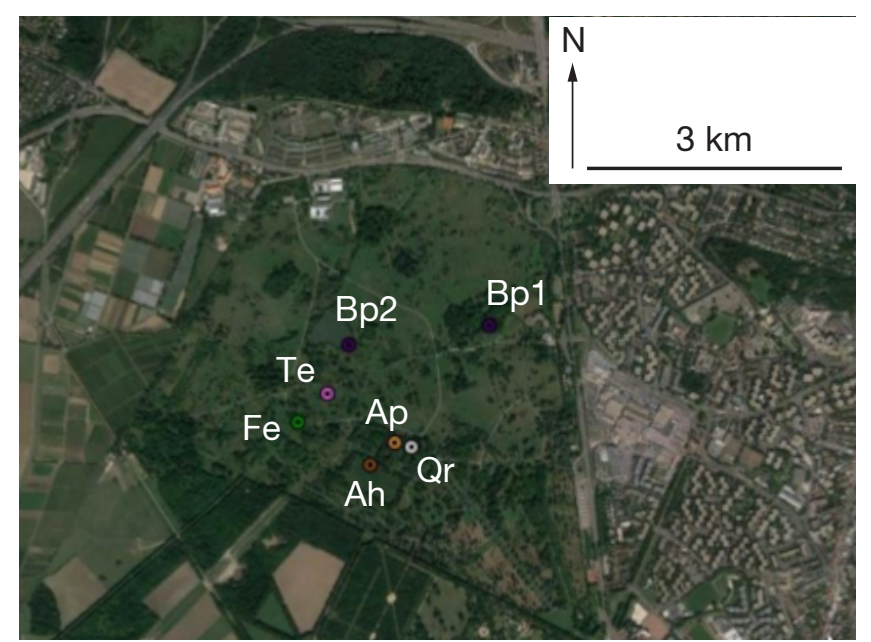

FIG. 2 - Localisation des essences d'arbres prospectées dans l'arboretum de Chèvreloup. Abréviations: Ah, Aesculus hippocastanum L. (Marronier d'Inde); Ap, Acer platanoides L. (Érable plane); Bp, Betula pendula Roth (Bouleau); Fe, Fraxinus excelsior L. (Frêne commun); Qr, Quercus robur L. (Chêne pédonculé); Te, Tilia $\times$ europaea L. (Tilleul). Un point correspond à cinq arbres, sauf $\mathrm{Bp} 1$ et $\mathrm{Bp} 2$ qui comportent trois arbres chacun. Vue satellitaire: GoogleEarth.

été plantés de manière groupée avec un espace régulier entre chaque arbre dans un sol granuleux mais moins émetteur de poussières que l'allée Gay-Lussac. Enfin, les cinq platanes situés dans l'allée avec le monument Delacroix, près de l'Orangerie du Sénat, ont été prospectés; le terrain calcaire est similaire à celui de l'allée Gay-Lussac où les marronniers ont été étudiés.

\section{L'arboretum de Chèvreloup}

L'arboretum de Versailles-Chèvreloup se situe dans le département des Yvelines, à $6 \mathrm{~km}$ de la ville de Versailles et $24 \mathrm{~km}$ à l'Ouest de la ville de Paris, et jouxte le parc de Trianon du château de Versailles.

Six espèces d'arbres ont été inventoriées: Marronnier d'Inde (Aesculus hippocastanum), Érable plane (Acer platanoides L.), Bouleau (Betula pendula Roth), Frêne commun (Fraxinus excelsior), Chêne pédonculé (Quercus robur L.) et Tilleul (Tilia $\times$ europaea L.) (Fig. 2).

Il n'a pas été possible de trouver cinq individus dans une même zone pour deux essences d'arbres. Ainsi trois individus de bouleau ont été prospectés près d'un étang («Bp $1 »)$ et trois autres plus loin, près d'un ruisseau («Bp $2 »)$ (Fig. 2). Quatre frênes ont été observés. Le site de Chèvreloup se distingue des sites parisiens par son substrat enherbé et son absence de taille des arbres.

\section{Protocole DE TERRAIN}

Deux types de protocoles de relevés de biodiversité ont été suivis. Le premier reprend la norme AFNOR (Association française de Normalisation) (NF EN 16413 2014) avec des grilles d'observation (quadrats), le second est adapté de la méthode IAP (Index of Atmospheric Purity) développée par LeBlanc \& Sloover 1970. Une prospection additionnelle a été réalisée sur les stations parisiennes.

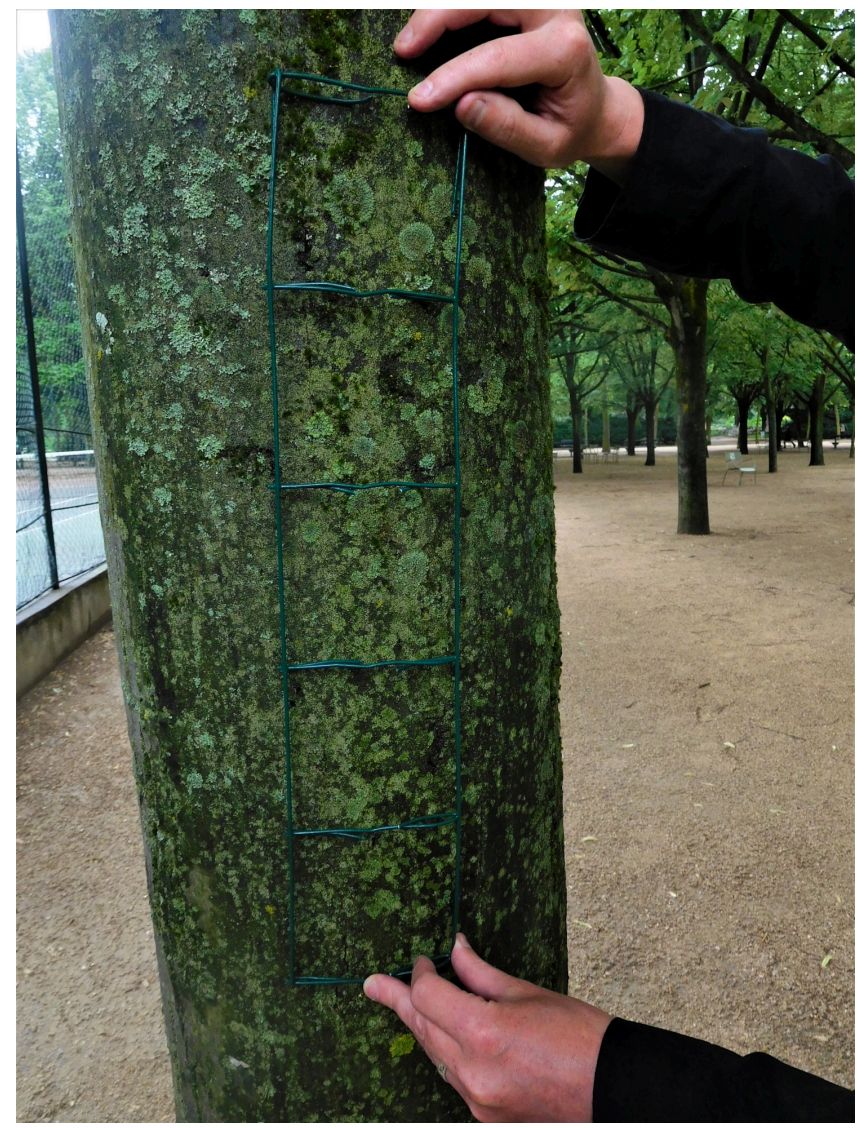

FIG. 3. - Grille d'observation placée sur un point cardinal du tronc d'un Tilleul, Tilia $\times$ europaea L., au jardin du Luxembourg. Crédit photo: É. Lebreton.

\section{Protocole AFNOR}

Les essences d'arbres ont été sélectionnées de sorte qu'il y ait trois à cinq phorophytes par essence sur chaque site mais également des essences communes entre sites. Les arbres d'alignement respectant les critères morphologiques de la norme AFNOR (NF EN 164132014 ) ont été sélectionnés : tronc droit (ni coupé ni fendu), circonférence comprise entre 50 et $250 \mathrm{~cm}$ et recouvrement bryophytique inférieur à $20 \%$. Les relevés lichéniques se font au moyen de grilles d'observation (Fig. 3).

Il s'agit d'une grille de $10 \times 50 \mathrm{~cm}$ divisée en cinq mailles de $10 \times 10 \mathrm{~cm}$. Les grilles sont placées sur le tronc aux quatre points cardinaux définis grâce à une boussole. La base de la grille, formée de cinq carrés de $10 \times 10 \mathrm{~cm}$, est positionnée à un mètre au-dessus de la base du tronc. La diversité taxonomique des lichens a été relevée sur une surface de $10 \mathrm{~cm}$ de largeur sur $50 \mathrm{~cm}$ de longueur pour chaque point cardinal de l'arbre.

\section{Protocole IAP}

Ce protocole reprend exactement les mêmes arbres que ceux observés dans le protocole AFNOR. La prospection des arbres est élargie au pourtour du tronc allant de la base du tronc jusqu'à $2 \mathrm{~m}$ de hauteur, telle qu'elle est préconisée dans le protocole IAP. Les lichens du protocole adapté de la méthode IAP s'interprètent comme étant la diversité taxono- 
mique trouvée par le protocole AFNOR associée à la diversité taxonomique trouvée sur la base des tronc (de 0 à $1 \mathrm{~m}$ ) et en haut des troncs de (1,5 à $2 \mathrm{~m})$.

\section{Prospection additionnelle}

Une trentaine d'arbres supplémentaires ont été également observés sur les sites du JDP et du JDL afin de compléter l'inventaire de biodiversité. Au jardin du Luxembourg, en plus des 20 phorophytes observés (Fig. 1), nous avons examiné l'ensemble des marronniers de l'allée de l'observatoire et de Gay Lussac, ainsi que les tilleuls entourant le terrain de tennis, et les platanes de l'allée longeant la rue Médicis. Nous avons également observé un Gingko, de jeunes arbres de Judée et des arbustes de la famille des Fabaceae qui venaient d'être plantés. Au jardin des Plantes, nous avons examiné l'ensemble des platanes localisés le long de l'allée jouxtant le bâtiment de l'herbier national, ainsi que le savonnier de l'allée Jussieu.

Les espèces de lichens dont la détermination sur arbre est possible ont été notées directement sur les fiches de relevé de terrain, tandis que les spécimens non-identifiables sur site ont été échantillonnés dans des sachets en papier pour une détermination en laboratoire, puis conservés dans l'herbier PC du MNHN. Les échantillons du JDL vont du code PC0779972 à PC0779923, ceux du BDS de PC0779973 à PC0779997 et ceux du JDP de PC0779922 à PC0779858. Les codes des échantillons de Chèvreloup ne sont pas disponibles actuellement.

\section{DÉTERMINATION DES LICHENS AU LABORATOIRE}

Les lichens ont été identifiés selon les méthodes standards de lichénologie (Coste 1991). Les critères morphologiques ont été étudiés avec une loupe binoculaire. L’observation des structures microscopiques (étude des différentes structures thallines et relatives aux ascomes, etc.) a été effectuée avec un microscope Nikon Eclipse 50i sur des coupes montées dans différents milieux: de l'eau, une solution aqueuse d'hydroxyde de potassium à $10 \%$, une solution iodo-iodurée: Lugol et/ou une solution de bleu coton lactophénolée selon les besoins.

La détermination des lichens a été effectuée principalement à partir des livres de Clauzade \& Roux 1985; Smith et al. 2009; Van Haluwyn \& Asta 2013; Wirth et al. 2013. D'autres documents ont été utilisés, tels que les fiches espèces et la bibliographie présente sur le site de l'AFL (Association française de Lichénologie: www.afl-lichenologie.fr, dernière consultation le 18 août 2018). La nomenclature retenue est celle du catalogue des lichens et champignons lichénicoles de France métropolitaine (Roux 2020).

\section{RÉSULTATS ET DISCUSSION}

\section{LISTE DES TAXONS}

Le Tableau 1 présente les taxons inventoriés sur les arbres d'alignement parisiens et les phorophytes de Chèvreloup avec les protocoles AFNOR et IAP.
La prospection d'arbres supplémentaires a permis d'ajouter quatre espèces pour Paris: Evernia prunastri (L.) Ach., Lecanora leptyrodes (Nyl.) Degel., Melanelixia glabratula (Lamy) Sandler \& Arup et Punctelia jeckeri (Roum.) Kalb. P. jeckeri a été trouvée sur un arbre de Judée au jardin du Luxembourg et L. leptyrodes sur un savonnier dans le jardin des Plantes. Appelé à tort "mousse du chêne», E. prunastri a été retrouvée sur un arbuste tout juste planté au jardin du Luxembourg. Cette dernière occurrence doit être prise avec précaution, car la présence de ce lichen pourrait être due à l'introduction de l'arbre issu d'une pépinière localisée hors de la métropole parisienne. Il est donc difficile de savoir si cette espèce pourra s'établir durablement sur ce site. De plus, ces arbres récemment plantés étaient les seuls à porter des spécimens de $M$. glabratula qui montraient des signes d'une dégénérescence (blanchiment du thalle). Les espèces $L$. leptyrodes et $M$. glabratula ont été observées avec les protocoles AFNOR et IAP sur le site de Chèvreloup.

L'étude des quatre sites a permis de dénombrer un total de 63 espèces de lichens: 61 espèces avec les protocoles AFNOR + IAP et deux espèces avec la prospection additionnelle. Ces taxons se répartissent en 14 familles et 35 genres. Parmi ces espèces, 13 espèces sont corticoles strictes, trois espèces sont saxicoles strictes et 47 espèces sont ubiquistes (Roux 2017). À Paris, 47 espèces de lichens ont été recensées (43 avec les protocoles AFNOR + IAP et quatre supplémentaires), et 43 espèces pour l'arboretum de Chèvreloup avec les protocoles AFNOR + IAP. Selon le catalogue des lichens de France (Roux 2017), cinq espèces sont nouvellement mentionnées pour la région Île-de-France (Caloplaca arnoldii (Wedd.) Zahlbr. ex Ginzb., Caloplaca flavocitrina (Nyl.) H. Olivier, Endocarpon adscendens (Anzi) Müll. Arg., Lecanora leptyrodes (Nyl.) Degel, Xanthomendoza huculica (S.Y.Kondr) Diederich) (Fig. 4A-H) et trois en plus pour la ville de Paris (Flavoparmelia soredians (Nyl.) Hale, Lecanora compallens van Herk \& Aptroot, Rinodina gennarii Bagl.).

Certaines découvertes peuvent être attribuées aux récents progrès de la lichénologie. En effet, L. leptyrodes a jusque récemment été confondu avec Lecanora carpinea (Van Haluwyn \& Asta 2013). La distinction s'effectue avec un examen microscopique des apothécies révélant chez $L$. leptyrodes un bord thallin à pseudocortex non gélatineux, riche en cristaux (petits ou moyens) insolubles dans le potassium. De plus, ces deux taxons peuvent coexister dans une même station voire sur un même arbre, ce qui peut rendre la confusion d'autant plus grande (Chantal Van Haluwyn comm. pers.). Le même constat peut être observé avec $L$. compallens, décrite en 1999, et longtemps confondue avec Lecanora expallens Ach. tout comme $X$. huculica qui a jusque très récemment été confondu avec Xanthoria fallax (Hepp) Arnold (Roux 2017). Il est donc probable que la révision de spécimens d'herbier collectés en Île-de-France remette à jour ces nouveautés.

Certaines espèces n'avaient pas été mentionnées depuis le XIXe siècle à Paris. La dernière mention de Toninia populorum (A. Massal.) Kistenich, Timdal, Bendiksby \& S. Ekman, Caloplaca cerinella (Nyl.) Flagey et Myriolecis crenulata (Ach.) Śliwa, Zhao Xin \& Lumbsch à Paris remonte au siècle dernier 
TABLEAU 1. - Taxons lichéniques inventoriés sur les arbres d'alignement parisiens et les phorophytes de Chèvreloup avec les protocoles AFNOR (Association française de Normalisation) et IAP (Index of Atmospheric Purity). Pour le protocole IAP, figurent dans le tableau uniquement les taxons non inventoriés par le protocole AFNOR. Abréviations: des stations, BDSB, bords de Seine station Bercy; BDSI, bord de Seine station Île aux Cygnes; BDSP, bord de Seine station Pont Neuf; CL, Chèvreloup; JDL, jardin du Luxembourg; JDP, jardin des plantes; des essences d'arbres, Ah, Aesculus hippocastanum L. (Marronier d'Inde); Ah1, Aesculus hippocastanum de l'allée de l'Observatoire; Ah2, Aesculus hippocastanum de l'allée de Gay-Lussac; Ap, Acer platanoides L. (Érable plane); Bp1, Betula pendula Roth (Bouleau) de la prairie; Bp2, Betula pendula de la mare; Fe, Fraxinus excelcior L. (Frêne commun); Ph, Platanus $\times$ hispanica Mill. ex Münchh. (Platane); Pi, Populus cf. nigra var. italica Du Roi (Peuplier noir); Pc, Populus $\times$ canescens (Aiton) Sm. (Peuplier grisard); Qr, Quercus robur L. (Chêne pédonculé); Te, Tilia × europaea L. (Tilleul); de l'écologie des lichens selon Roux et al. (2017), Cort., corticole, qui se développe sur écorce; Fol., foliicole, qui se développe sur feuille; Lign., lignicole, qui se développe sur bois; Musc., muscicole, qui se développe sur les mousses; Sax., saxicole, qui se développe sur roche; Ter., terricole, qui se développe sur le sol; sans les parenthèses, lichen habituellement observé sur ce substrat; avec les parenthèses, lichen rarement/exceptionnellement observé sur ce substrat.

\begin{tabular}{|c|c|c|c|c|c|c|c|c|c|c|c|c|c|}
\hline \multirow[b]{3}{*}{ Taxons } & \multicolumn{12}{|c|}{ Stations et protocoles } & \multirow[b]{3}{*}{ Écologie } \\
\hline & \multicolumn{2}{|l|}{ CL } & \multicolumn{2}{|c|}{ BDSB } & \multicolumn{2}{|c|}{ BDSI } & \multicolumn{2}{|c|}{ BDSP } & \multicolumn{2}{|c|}{ JDL } & \multicolumn{2}{|c|}{ JDP } & \\
\hline & AFNOR & IAP & AFNOR & IAP & AFNOR & IAP & AFNOR & IAP & AFNOR & IAP & AFNOR & IAP & \\
\hline \multicolumn{14}{|l|}{ Lichens } \\
\hline $\begin{array}{l}\text { Alyxoria varia (Pers.) Ertz \& } \\
\text { Tehler }\end{array}$ & $\mathrm{Fe}$ & - & - & - & - & - & - & - & - & - & - & - & Cort. (Sax.) \\
\hline $\begin{array}{l}\text { Amandinea punctata (Hoffm.) } \\
\text { Coppins \& Scheid. }\end{array}$ & $\begin{array}{c}\text { Ah Ap Bp1 } \\
\text { Bp2 Fe } \\
\text { Qr Te }\end{array}$ & - & - & - & Ah & - & - & - & Ah1 Ah2 & $\mathrm{Te}$ & $\mathrm{Ah} \mathrm{Fe}$ & - & $\begin{array}{l}\text { Cort. (Sax.) } \\
\text { (Terr.) }\end{array}$ \\
\hline $\begin{array}{l}\text { Arthonia atra (Pers.) A. } \\
\text { Schneid. }\end{array}$ & - & Bp1 & - & - & - & - & - & - & - & - & - & - & Cort. \\
\hline $\begin{array}{l}\text { Bacidia arceutina (Ach.) } \\
\quad \text { Arnold }\end{array}$ & $\mathrm{Te}$ & - & - & - & - & - & - & - & - & - & - & - & $\begin{array}{l}\text { Cort. (Sax.) } \\
\text { (Musc.) }\end{array}$ \\
\hline $\begin{array}{l}\text { Bacidia laurocerasi (Delise ex } \\
\text { Duby) Zahlbr. }\end{array}$ & $\mathrm{Fe}$ & - & - & - & - & - & - & - & - & - & - & - & $\begin{array}{l}\text { Cort. (Lign.) } \\
\text { (Fol.) }\end{array}$ \\
\hline $\begin{array}{l}\text { Caloplaca arnoldii (Wedd.) } \\
\text { Zahlbr. ex Ginzb. }\end{array}$ & - & - & - & - & - & - & - & - & - & Ah2 & - & - & Sax. \\
\hline $\begin{array}{l}\text { Caloplaca cerinella (Nyl.) } \\
\text { Flagey }\end{array}$ & - & - & $\mathrm{PcPi}$ & - & - & - & - & - & - & $\mathrm{Ph}$ & $\mathrm{Ah} \mathrm{Ph}$ & - & Cort. \\
\hline $\begin{array}{l}\text { Caloplaca citrina (Hoffm.) } \\
\text { Th. Fr. }\end{array}$ & - & - & - & - & - & - & - & - & - & Ah2 & - & $\mathrm{Ph}$ & $\begin{array}{l}\text { Sax. (Cort.) } \\
\quad \text { (Lign.) }\end{array}$ \\
\hline $\begin{array}{l}\text { Caloplaca flavocitrina (Nyl.) } \\
\text { H. Olivier }\end{array}$ & - & - & - & - & Ah & - & - & - & - & Ah2 & - & $\mathrm{Fe}$ & $\begin{array}{l}\text { Sax. (Cort.) } \\
\text { (Lign.) }\end{array}$ \\
\hline Caloplaca groupe citrina & - & - & - & - & Ah & - & - & - & - & Ah1 & - & Ah Fe Ph & - \\
\hline $\begin{array}{l}\text { Caloplaca pyraceae (Ach.) } \\
\text { Th. Fr }\end{array}$ & - & - & - & - & - & - & - & - & Ah2 & $\mathrm{Ph}$ & $\mathrm{Ph}$ & - & Cort. (Lign.) \\
\hline $\begin{array}{l}\text { Candelaria concolor (Dicks.) } \\
\text { Stein }\end{array}$ & $\begin{array}{c}\text { Ap Bp1 Bp2 } \\
\text { Qr Te }\end{array}$ & $\mathrm{Fe}$ & - & - & Ah & - & - & - & $\begin{array}{c}\text { Ah1 } \mathrm{Ph} \\
\mathrm{Te}\end{array}$ & - & $\mathrm{Ah} \mathrm{Fe}$ & - & $\begin{array}{l}\text { Cort. (Sax.) } \\
\quad \text { (Lign.) }\end{array}$ \\
\hline $\begin{array}{l}\text { Candelariella aurella (Hoffm.) } \\
\text { Zahlbr. }\end{array}$ & - & - & - & - & - & - & - & - & - & Ah2 & - & - & Sax. \\
\hline Candelariella sp.1 & - & Bp2 & - & - & - & - & - & - & - & - & - & - & - \\
\hline $\begin{array}{l}\text { Candelariella xanthostigma } \\
\text { (Ach.) Lettau }\end{array}$ & $\begin{array}{c}\text { Ap Bp1 Bp2 } \\
\text { Fe Qr Te }\end{array}$ & - & $\mathrm{PcPi}$ & - & Ah & - & - & - & - & Ah1 Te & Ah & - & Cort. (Lign.) \\
\hline $\begin{array}{l}\text { Catillaria nigroclavata (Nyl.) } \\
\text { Schuler }\end{array}$ & $\mathrm{Te}$ & - & - & - & - & - & - & - & - & - & - & - & Cort. (Lign.) \\
\hline $\begin{array}{l}\text { Coenogonium pineti (Schrad. } \\
\text { ex Ach.) Lücking \& } \\
\text { Lumbsch }\end{array}$ & Ah Bp1 & - & - & - & - & - & - & - & - & - & - & - & $\begin{array}{l}\text { Cort. (Lign.) } \\
\text { (Fol.) } \\
\text { (Sax.) }\end{array}$ \\
\hline $\begin{array}{l}\text { Diploicia canescens (Dicks.) } \\
\text { A. Massal }\end{array}$ & - & Ap Qr & - & - & - & - & - & - & - & Ah1 & - & Ah & $\begin{array}{l}\text { Sax. Cort. } \\
\text { (Musc.) }\end{array}$ \\
\hline $\begin{array}{l}\text { Endocarpon adscendens } \\
\text { (Anzi) Müll. Arg. }\end{array}$ & - & - & - & - & - & - & - & - & - & - & - & $\mathrm{Fe}$ & $\begin{array}{l}\text { Sax. Terr. } \\
\text { Musc. }\end{array}$ \\
\hline $\begin{array}{l}\text { Flavoparmelia caperata (L.) } \\
\quad \text { Hale }\end{array}$ & Bp1 Bp2 Te & Qr & - & - & - & - & - & - & $\mathrm{Te}$ & - & - & - & Cort. (Sax.) \\
\hline $\begin{array}{l}\text { Flavoparmelia soredians (Nyl.) } \\
\text { Hale }\end{array}$ & - & - & - & - & - & - & - & - & - & $\mathrm{Te}$ & - & $\mathrm{Ah}$ & Cort. (Sax.) \\
\hline $\begin{array}{l}\text { Hyperphyscia adglutinata } \\
\text { (Flörke) H. Mayrhofer \& } \\
\text { Poelt }\end{array}$ & $\begin{array}{c}\text { Ah Ap Bp1 } \\
\text { Bp2 Qr } \\
\text { Te }\end{array}$ & - & $\mathrm{Pc} \mathrm{Pi}$ & - & Ah & - & Ah & - & $\begin{array}{l}\text { Ah1 Ah2 } \\
\text { Ph Te }\end{array}$ & - & $\mathrm{Ah} \mathrm{Fe} \mathrm{Ph}$ & - & $\begin{array}{l}\text { Cort. (Lign.) } \\
\quad \text { (Fol.) } \\
\text { (Sax.) }\end{array}$ \\
\hline $\begin{array}{l}\text { Hypogymnia physodes (L.) } \\
\text { Nyl. }\end{array}$ & - & Ap & - & - & - & - & - & - & - & - & - & - & $\begin{array}{l}\text { Cort. Lign. } \\
\text { (Sax.) } \\
\text { (Musc.) }\end{array}$ \\
\hline Lecania cyrtella (Ach.) Th. Fr. & Ah Ap Bp2 & - & - & - & - & - & - & - & - & - & $\mathrm{Ph}$ & Ah Fe & Cort. \\
\hline Lecania sp. & - & - & $\mathrm{Pc}$ & - & - & - & - & - & - & - & - & Ah & - \\
\hline $\begin{array}{l}\text { Lecania naegelii (Hepp) } \\
\text { Diederich \& van den Boom }\end{array}$ & $\mathrm{Bp} 2 \mathrm{Fe} \mathrm{Te}$ & Ah Ap & - & - & - & - & - & - & - & - & - & - & $\begin{array}{l}\text { Cort. (Lign.) } \\
\text { (Sax.) }\end{array}$ \\
\hline Lecanora allophana (Ach.) Nyl. & - & $\mathrm{Ah}$ & - & - & - & - & - & - & - & - & - & - & Cort. \\
\hline $\begin{array}{l}\text { Lecanora argentata (Ach.) } \\
\quad \text { Malme }\end{array}$ & Ap & - & - & - & - & - & - & - & - & - & - & - & Cort. \\
\hline Lecanora carpinea (L.) Vain. & $\mathrm{Te}$ & Bp2 & - & - & - & - & - & - & - & Ah1 & - & - & Cort. (Lign.) \\
\hline Lecanora chlarotera Nyl. & $\begin{array}{c}\text { Ah Ap Bp1 } \\
\text { Bp2 Fe } \\
\text { Te }\end{array}$ & - & - & - & - & - & - & - & $\mathrm{Te}$ & - & - & Ah & Cort. \\
\hline $\begin{array}{l}\text { Lecanora compallens van } \\
\text { Herk \& Aptroot }\end{array}$ & $\begin{array}{c}\text { Ah Ap Bp1 } \\
\text { Bp2 Fe } \\
\text { Qr }\end{array}$ & - & - & - & Ah & - & - & - & $\mathrm{Te}$ & - & - & Ah & Cort. \\
\hline $\begin{array}{l}\text { Lecanora conizaeoides Nyl. ex } \\
\text { Cromb. }\end{array}$ & Qr Te & $\mathrm{Fe}$ & - & - & Ah & - & - & - & - & - & - & - & $\begin{array}{l}\text { Cort. (Lign.) } \\
\quad \text { (Sax.) }\end{array}$ \\
\hline
\end{tabular}


TABLEAU 1. - Suite.

\begin{tabular}{|c|c|c|c|c|c|c|c|c|c|c|c|c|c|}
\hline \multirow[b]{3}{*}{ Taxons } & \multicolumn{12}{|c|}{ Stations et protocoles } & \multirow[b]{3}{*}{ Écologie } \\
\hline & \multicolumn{2}{|l|}{$\mathrm{CL}$} & \multicolumn{2}{|c|}{ BDSB } & \multicolumn{2}{|c|}{ BDSI } & \multicolumn{2}{|c|}{ BDSP } & \multicolumn{2}{|c|}{ JDL } & \multicolumn{2}{|c|}{ JDP } & \\
\hline & AFNOR & IAP & AFNOR & IAP & AFNOR & IAP & AFNOR & IAP & AFNOR & IAP & AFNOR & IAP & \\
\hline Lecanora horiza (Ach.) Linds. & Bp2 & - & - & - & - & - & - & - & - & - & - & - & Cort. \\
\hline $\begin{array}{l}\text { Lecanora leptyrodes (Nyl.) } \\
\text { Degel. }\end{array}$ & Bp2 & - & - & - & - & - & - & - & - & - & - & - & - \\
\hline Lecanora sp. & - & Ah & - & - & - & - & - & - & - & - & - & - & - \\
\hline $\begin{array}{l}\text { Lecidella elaeochroma (Ach.) } \\
\text { M.Choisy }\end{array}$ & $\begin{array}{c}\text { Ah Ap Bp2 } \\
\text { Fe Te }\end{array}$ & Bp1 & - & - & Ah & - & - & - & Ah1 & - & - & Ah & Cort. (Lign.) \\
\hline Lepraria incana (L.) Ach. & $\begin{array}{l}\text { Ah Ap Bp1 } \\
\text { Fe Qr Te }\end{array}$ & - & - & - & - & - & - & - & - & $\mathrm{Te}$ & Ah & - & $\begin{array}{l}\text { Cort. (Lign.) } \\
\text { (Sax.) } \\
\text { (Ter.) }\end{array}$ \\
\hline $\begin{array}{l}\text { Melanelixia glabratula (Lamy) } \\
\text { Sandler \& Arup }\end{array}$ & Bp1 & - & - & - & - & - & - & - & - & - & - & - & $\begin{array}{l}\text { Cort. (Lign.) } \\
\text { (Sax.) }\end{array}$ \\
\hline $\begin{array}{l}\text { Melanelixia subaurifera (Nyl.) } \\
\text { O. Blanco, A. Crespo, } \\
\text { Divakar, Essl., D. Hawksw. } \\
\text { \& Lumbsch }\end{array}$ & Bp1 & - & - & - & - & - & - & - & - & $\mathrm{Te}$ & - & - & $\begin{array}{l}\text { Cort. (Lign.) } \\
\text { (Sax.) } \\
\text { (Musc.) }\end{array}$ \\
\hline $\begin{array}{l}\text { Myriolecis albescens (Hoffm.) } \\
\text { Śliwa, Zhao Xin \& Lumbsch }\end{array}$ & - & - & - & - & - & - & - & - & - & Ah2 & - & $\mathrm{Fe} \mathrm{Ph}$ & Sax. (Lign.) \\
\hline $\begin{array}{l}\text { Myriolecis crenulata (Hook.) } \\
\text { Śliwa, Zhao Xin \& Lumbsch }\end{array}$ & - & - & - & - & - & - & - & - & - & - & $\mathrm{Ph}$ & - & Sax. \\
\hline $\begin{array}{l}\text { Myriolecis dispersa (Pers.) } \\
\text { Śliwa, Zhao Xin et Lumbsch }\end{array}$ & - & - & - & - & Ah & - & - & - & - & Ah2 Te & Ah & - & Sax. (Lign.) \\
\hline $\begin{array}{l}\text { Myriolecis hagenii (Ach.) } \\
\text { Śliwa, Zhao Xin et Lumbsch }\end{array}$ & Bp2 & $\mathrm{Te}$ & $\mathrm{Pi}$ & - & - & - & - & - & - & Ah2 Ah1 & Ah & $\mathrm{Fe} P \mathrm{Ph}$ & $\begin{array}{l}\text { Cort. (Lign.) } \\
\text { (Fol.) } \\
\text { (Sax.) }\end{array}$ \\
\hline Parmelia sulcata Taylor s.l. & $\begin{array}{l}\mathrm{Bp} 1 \mathrm{Bp} 2 \mathrm{Fe} \\
\text { Qr Te }\end{array}$ & - & - & - & Ah & - & - & - & - & $\mathrm{Te}$ & - & - & $\begin{array}{l}\text { Cort. (Lign.) } \\
\text { (Sax.) }\end{array}$ \\
\hline $\begin{array}{l}\text { Parmotrema perlatum (Huds.) } \\
\text { M. Choisy }\end{array}$ & $\begin{array}{c}\text { Bp1 Bp2 Qr } \\
\text { Te }\end{array}$ & $\mathrm{Fe}$ & - & - & - & - & - & - & $\mathrm{Te}$ & - & - & $\mathrm{Fe}$ & $\begin{array}{l}\text { Cort. (Lign.) } \\
\text { (Sax.) } \\
\text { (Musc.) }\end{array}$ \\
\hline $\begin{array}{l}\text { Phaeophyscia orbicularis } \\
\text { (Neck.) Moberg }\end{array}$ & Bp1 Bp2 Te & - & $\mathrm{Pc} \mathrm{Pi}$ & - & Ah & - & - & - & Ah1 Te & $\mathrm{Ph}$ & $\mathrm{Ah} \mathrm{Fe} \mathrm{Ph}$ & - & $\begin{array}{l}\text { Cort. (Sax.) } \\
\quad \text { (Lign.) } \\
\text { (Musc.) }\end{array}$ \\
\hline Phlyctis argena (Spreng.) Flot & $\mathrm{Te}$ & Qr & - & - & - & - & - & - & - & - & - & - & Cort. (Sax.) \\
\hline Physcia adscendens H.Olivier & Bp1 Bp2 & - & $\mathrm{Pc} \mathrm{Pi}$ & - & Ah & - & Ah & - & $\mathrm{Te}$ & $\mathrm{Ph}$ & Ah Fe Ph & - & $\begin{array}{l}\text { Cort. (Sax.) } \\
\text { (Lign.) } \\
\text { (Fol.) }\end{array}$ \\
\hline Physcia tenella (Scop.) DC & $\begin{array}{c}\text { Ah Ap Bp1 } \\
\text { Bp2 Fe } \\
\text { Te }\end{array}$ & - & $\mathrm{Pc} \mathrm{Pi}$ & - & Ah & - & - & - & $\begin{array}{l}\text { Ah1 } \mathrm{Ph} \\
\mathrm{Te}\end{array}$ & Ah2 & $\mathrm{Ah} \mathrm{Fe} \mathrm{Ph}$ & - & $\begin{array}{l}\text { Cort. (Lign.) } \\
\quad \text { (Sax.) }\end{array}$ \\
\hline $\begin{array}{l}\text { Physconia grisea subsp. } \\
\text { grisea (Lam.) Poelt }\end{array}$ & Bp2 Te & - & $\mathrm{Pi}$ & - & - & - & - & - & Ah1 & - & $\mathrm{Ah} \mathrm{Fe}$ & - & $\begin{array}{l}\text { Cort. (Lign.) } \\
\text { (Sax.) }\end{array}$ \\
\hline $\begin{array}{l}\text { Physconia perisidiosa } \\
\text { (Erichsen) Moberg }\end{array}$ & - & $\mathrm{Bp} 2 \mathrm{Te}$ & - & - & - & - & - & - & - & - & - & - & Cort. (Sax.) \\
\hline $\begin{array}{l}\text { Pleurosticta acetabulum } \\
\text { (Neck.) Elix \& Lumbsch }\end{array}$ & Bp2 Qr & Bp1 & - & - & Ah & - & - & - & - & - & - & - & Cort. \\
\hline $\begin{array}{l}\text { Protoparmeliopsis muralis } \\
\text { (Schreb.) M. Choisy }\end{array}$ & - & - & - & - & - & - & - & - & - & - & - & Ah & Sax. (Lign.) \\
\hline $\begin{array}{l}\text { Pseudoschismatomma } \\
\text { rufescens (Pers.) Ertz \& } \\
\text { Tehler }\end{array}$ & Ah & - & - & - & - & - & - & - & - & Ah1 & - & - & Cort. \\
\hline Punctelia borreri (Sm.) Krog & - & Вp2 & - & - & Ah & - & - & - & $\mathrm{Te}$ & - & - & $\mathrm{Fe}$ & Cort. Sax. \\
\hline $\begin{array}{l}\text { Punctelia subrudecta (Nyl.) } \\
\text { Krog }\end{array}$ & - & - & - & - & Ah & - & - & - & $\mathrm{Te}$ & - & - & $\mathrm{Fe}$ & Cort. \\
\hline Ramalina farinacea (L.) Ach. & Bp2 & - & - & - & - & - & - & - & - & - & Ah & - & $\begin{array}{l}\text { Cort. (Lign.) } \\
\text { (Sax.) } \\
\text { (Ter.) }\end{array}$ \\
\hline Rinodina gennarii Bagl. & - & - & - & - & - & - & - & - & - & Ah2 & Ah & - & Sax. (Lign.) \\
\hline $\begin{array}{l}\text { Toninia populorum (A. } \\
\text { Massal.) Kistenich, Timdal, } \\
\text { Bendiksby \& S. Ekman }\end{array}$ & - & - & $\mathrm{Pi}$ & - & & - & - & - & - & - & - & - & Cort. (Sax.) \\
\hline $\begin{array}{l}\text { Xanthomendoza huculica } \\
\text { (S.Y.Kondr) Diederich }\end{array}$ & - & - & - & - & - & - & - & - & - & $\mathrm{Te}$ & - & - & Cort. \\
\hline $\begin{array}{l}\text { Xanthoria parietina var. } \\
\text { parietina (L.) Th. Fr. }\end{array}$ & $\begin{array}{c}\text { Ap Bp1 Bp2 } \\
\text { Fe Qr Te }\end{array}$ & - & $\mathrm{PcPi}$ & - & Ah & - & Ah & - & $\begin{array}{r}\text { Ah1 Ah2 } \\
\text { Ph Te }\end{array}$ & - & Ah Fe Ph & - & $\begin{array}{l}\text { Cort. Sax. } \\
\text { Lign. }\end{array}$ \\
\hline \multicolumn{14}{|l|}{ Myxomycètes } \\
\hline $\begin{array}{l}\text { Licea parasitica (Zukal) } \\
\text { G.W.Martin }\end{array}$ & - & - & $\mathrm{Pi}^{\star}$ & - & - & - & - & - & - & - & - & - & - \\
\hline \multicolumn{14}{|l|}{ Algues } \\
\hline Pleuroccoccus sp. & $\begin{array}{c}\text { Ap Bp1 Bp2 } \\
\text { Fe Te }\end{array}$ & - & $\mathrm{PcPi}$ & - & Ah & - & Ah & - & $\begin{array}{l}\text { Ah1 Ah2 } \\
\text { Ph Te }\end{array}$ & - & $\mathrm{Ah} \mathrm{Fe} \mathrm{Ph}$ & - & - \\
\hline $\begin{array}{l}\text { Champignons non lichénisés } \\
\text { Hystérium sp. }\end{array}$ & - & - & - & - & - & - & - & - & - & Ah1 Ah2 & - & - & - \\
\hline
\end{tabular}




\begin{tabular}{|c|c|c|c|c|c|c|c|c|c|c|c|c|c|}
\hline \multirow[b]{3}{*}{ Taxons } & \multicolumn{12}{|c|}{ Stations et protocoles } & \multirow[b]{3}{*}{ Écologie } \\
\hline & \multicolumn{2}{|c|}{ CL } & \multicolumn{2}{|c|}{ BDSB } & \multicolumn{2}{|c|}{ BDSI } & \multicolumn{2}{|c|}{ BDSP } & \multicolumn{2}{|c|}{ JDL } & \multicolumn{2}{|c|}{ JDP } & \\
\hline & AFNOR & IAP & AFNOR & IAP & AFNOR & IAP & AFNOR & IAP & AFNOR & IAP & AFNOR & IAP & \\
\hline Mycète sp.1 & - & - & - & - & - & - & - & - & - & - & - & Ah & - \\
\hline Mycète sp.2 & - & - & - & - & - & - & - & - & - & - & - & Ah & - \\
\hline Mycète sp.3 & - & - & - & - & - & - & - & - & - & - & - & - & - \\
\hline Mycosporum sp. & - & - & - & - & - & - & - & - & - & Ah2 & - & - & - \\
\hline
\end{tabular}

lorsqu'elles avaient été identifiées par Nylander (Nylander 1866) (Fig. 4H). M. crenulata et $C$. cerinella, espèces fréquentes dans notre inventaire, ne figuraient pas dans les listes d'espèces recherchées à Paris avant 1993. De plus, ces espèces sont difficilement identifiables: $M$. crenulata est habituellement observée sur roches calcaires (Nylander 1896 avait observé l'espèce sur des murs calcaires à Paris en 1890) et C. cerinella ne dépasse guère $1 \mathrm{~mm}$ d'envergure.

T. populorum est une espèce aérohygrophile souvent observée dans les ripisylves et sa présence sur un peuplier des bords de Seine pourrait être due à l'humidité permanente engendrée par la proximité de la Seine et les crues hivernales. En France, $T$. populorum et $E$. adsendens sont des espèces rares qui sont jugées potentiellement menacées par Roux (2017). La présence de ces espèces dans Paris démontre l'intérêt d'étudier les écosystèmes urbains, considérés à tort comme des milieux à faible biodiversité (UICN France 2013).

\section{APPORT DES DIFFÉRENTS PROTOCOLES DE TERRAIN}

L'utilisation de trois méthodes d'inventaire a permis d'obtenir des listes de taxons complémentaires. À Chèvreloup, sur les 43 espèces inventoriées, 35 ont été trouvées avec le protocole AFNOR soit $81 \%$ des taxons retrouvés. La prospection du bas des arbres et des zones non couvertes par les grilles a permis d'ajouter huit espèces à l'inventaire. Les trois sites parisiens présentent des résultats contrastés. Pour les trois stations des bords de Seine, le protocole AFNOR permet d'inventorier l'ensemble des taxons présents (pour BDSB $\mathrm{N}=11$; pour BDSI $\mathrm{N}=18$; pour BDSP $\mathrm{N}=3$ ). Au jardin des Plantes, 33 espèces ont été inventoriées, 18 taxons avec le protocole AFNOR et 15 taxons avec le protocole IAP. Au jardin du Luxembourg, 35 espèces ont été inventoriées, 16 taxons avec le protocole AFNOR et 19 taxons avec le protocole IAP.

Le protocole AFNOR permet d'inventorier de $100 \%$ à $81 \%$ des lichens des stations du bord de Seine et de Chèvreloup. Le pourcentage tombe à $55 \%$ et $46 \%$ pour les sites du jardin des Plantes et du jardin du Luxembourg. Cette différence s'explique par une différence du cortège lichénique colonisant le bas et le haut des arbres dans les deux jardins parisiens, probablement dû aux conditions environnementales proche des arbres (substrat).

\section{DisTRIBUTION SPATIALE DES LICHENS}

L'étude du Tableau 1 tend à montrer une distribution spatialisée des lichens corticoles entre les quatre sites d'échantillonnages. Des études statistiques complèteront ces résultats et feront l'objet de publications futures.
Le site des bords de Seine est divisé en trois stations. Ce site présente 23 espèces de lichens mais seulement trois espèces sont communes à toutes les stations (Hyperphyscia adglutinata (Florke) H. Mayrhofer \& Poelt; Physcia adscendens (Fr.) Oliv; Xanthoria parietina var. parietina (L.) Th. Fr.). Il s'agit des trois uniques espèces observées à la station Pont-Neuf. Bien que présentant des similitudes en termes de conditions environnementales, les stations Bercy et Pont Neuf sont différentes en nombre d'espèces inventoriées. Cette différence de cortège peut être due aux essences d'arbres prospectées (dix peupliers à Bercy contre cinq marronniers à Pont-Neuf). Les stations Bercy et Île aux Cygnes possèdent six espèces en commun soit $26 \%$ des espèces observées sur l'ensemble des deux stations.

Les lichens du jardin des Plantes et du jardin du Luxembourg sont proches en termes de nombre d'espèces mais aussi de composition du cortège lichénique. En effet, avec les protocoles AFNOR + IAP, nous retrouvons 27 espèces communes entre les deux parcs soit $66 \%$ des espèces observées sur l'ensemble des deux jardins. Sur les 23 espèces inventoriées sur les bords de Seine, 19 espèces sont communes avec le jardin des Plantes (soit $51 \%$ des espèces observées sur l'ensemble des deux sites) et le jardin du Luxembourg (soit $49 \%$ des espèces observées sur l'ensemble des deux sites). Sur les 43 espèces de lichens relevés sur les trois sites parisiens, 18 espèces sont communes aux trois sites.

Le nombre d'espèces relevé avec les protocoles AFNOR et IAP est similaire entre Paris et Chèvreloup $(n=43)$. Au total, 25 espèces communes ont été inventoriées entre les deux sites d'étude, soit $41 \%$ des espèces présentes sur l'ensemble des deux sites. Dix-neuf espèces sont communes entre Chèvreloup et le jardin des Plantes, 22 espèces entre Chèvreloup et le jardin du Luxembourg et 16 espèces entre Chèvreloup et les bords de Seine.

La distribution spatiale des lichens corticoles en milieu urbain est un sujet d'étude complexe et multifactoriel (Llewellyn et al. 2020). Le revêtement du sol des parcs pourrait expliquer cette répartition particulière des lichens sur le tronc des arbres parisiens. En effet, des espèces saxicoles, colonisant habituellement les roches calcaires (Roux 2017), ont été observées sur écorce au jardin des plantes et au jardin du Luxembourg (Tableau 1). En détail, nous avons observé trois espèces saxicoles strictes (Caloplaca arnoldii, Candelariella aurella (Hoffm.) Zahlbr. et Myriolecis crenulata et huit préférentiellement saxicoles (Caloplaca citrina (Hoffm.) Th. Fr., C. flavocitrina, Diploicia canescens (Dicks.) A. Massal, E. adscendens, Myriolecis albescens (Hoffm.) Śliwa, Zhao Xin 

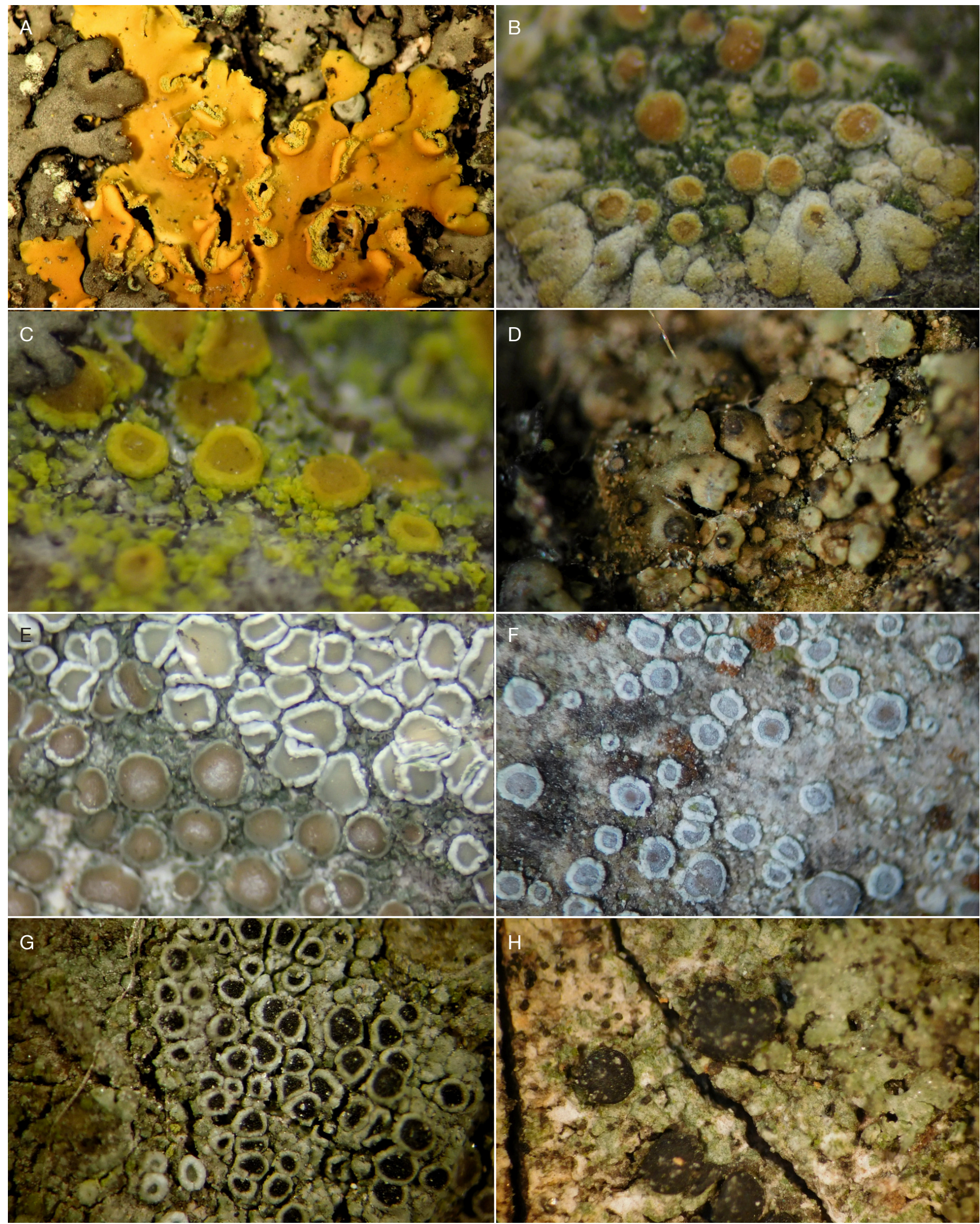

FIG. 4. - Photographies de huit espèces de lichens colonisant les arbres de Paris, vus à la loupe binoculaire. A, *Xanthomendoza huculica (S.Y.Kondr) Diederich; B, *Caloplaca arnoldii (Wedd.) Zahlbr. ex Ginzb.; C, Candelariella aurella (Hoffm.) Zahlbr.; D, *Endocarpon adsendens (Anzi) Müll. Arg.; E, Myriolecis albescens (Hoffm.) Śliwa, Zhao Xin \& Lumbsch; F, Myriolecis crenulata (Ach.) Śliwa, Zhao Xin \& Lumbsch; G, *Rinodina genarii Bagl.; H, Toninia populorum A. Massal.;

*, lichens nouveaux pour l'île-de-France. Crédits photos: C. Van Haluwyn (A, B), É. Lebreton (C-H). 
\& Lumbsch, Myriolecis dispersa (Pers.) Śliwa, Zhao Xin et Lumbsch, Protoparmeliopsis muralis (Schreb.) M. Choisy et $R$. gennarii). Ces lichens se trouvent exclusivement au pied des arbres, dans les premiers $20 \mathrm{~cm}$ du tronc. Avec le vent et le piétinement des visiteurs, ces deux sites sont caractérisés par une mise en suspension continuelle des poussières du revêtement des allées. Ces particules d'origine calcaire se déposent sur les troncs d'arbres par temps sec ou sont projetées en bas de l'arbre lors des pluies, ce qui favorise l'apparition de lichens saxicoles. L'écologie de ces espèces, essentiellement calcicoles, vient confirmer le rôle que jouent ces poussières sur l'écorce des arbres et sur la biodiversité. Au jardin du Luxembourg, le cortège lichénique diffère selon l'emplacement des marronniers, sur sol argileux (Ah1) ou sur sol calcaire (Ah2). Les marronniers poussiéreux du sol calcaire ne présentent plus de lichens au-delà de $1 \mathrm{~m}$ de hauteur, concentrant ainsi l'essentiel des espèces (saxicoles) en bas du tronc. Le phénomène inverse est observé sur les marronniers du sol argileux avec un cortège lichénique, colonisant préférentiellement le haut du tronc à partir de $1 \mathrm{~m}$, avec essentiellement des taxons corticoles. Les marronniers $\mathrm{du}$ jardin des Plantes profitent de deux effets combinés que sont l'humidité et l'empoussièrement. En effet, l'humidité engendrée par l'arrosage régulier des bandes herbeuses assure une colonisation des troncs en hauteur, tandis que la présence de poussière dans les allées permet l'apparition d'espèces saxicoles en bas de l'arbre. D'autres études plus spécifiques de l'effet de l'empoussièrement des bas de troncs doivent être menées pour valider statistiquement cette nouvelle niche écologique sur tronc. Il est donc important, dans un contexte stationnel particulier, de prendre en compte le bas des arbres comme niche supplémentaire dans les inventaires de biodiversité.

L'hypothèse «effet revêtement du sol » pourrait expliquer les différences de communautés lichéniques entre les sites parisiens et Chèvreloup. Les bas d'arbres prospectés à Chèvreloup présentent moins d'espèces de lichens que les arbres parisiens. De plus, le groupe d'espèces retrouvé dans ce site ne comporte pas d'espèces saxicoles, habituellement retrouvées sur roche. À Chèvreloup, les arbres croissent dans des pelouses plus ou moins hautes, tandis qu'à Paris les arbres sont soumis à l'empoussièrement.

Cet effet n'est pas visible pour les trois stations de bord de Seine où le protocole AFNOR a permis d'inventorier $100 \%$ des lichens présents sur les arbres. Peu d'espèces ont été trouvées sur le bas des arbres en raison des crues qui laissent de la boue sur les troncs, limitant ainsi le développement des lichens. De plus la présence des chiens, autorisés sur ces stations, implique l'existence d'une «zone canine» sur le bas des troncs rendant leur colonisation difficile par les lichens.

\section{CONCLUSION ET PERSPECTIVES}

Les données issues de nos inventaires ont été compilées dans une base de données qui sera mise en ligne et partagée sur l'Inventaire national du Patrimoine naturel (INPN). Soixante- trois taxons, dont plusieurs nouveautés écologiques comme des lichens saxicoles stricts sur arbre, ont été observés et ont déjà fait l'objet de notes dans le dernier catalogue des lichens de France (Roux 2020). Les 137 échantillons parisiens collectés dans le cadre de ces inventaires ont été intégrés à l'herbier (PC).

L'utilisation de trois protocoles complémentaires dans ces inventaires a permis de mettre en évidence une niche écologique méconnue, en l'absence de présence canine, qui se situe à la base du tronc des arbres. La mise en suspension des poussières issues du revêtement des sols des jardins parisiens permet aux lichens saxicoles (et plus particulièrement aux lichens calcicoles) d'étendre leur niche écologique et de se développer à la base des troncs.

L'existence de cette niche devra être confirmée par d'autres inventaires en milieu urbain, mais également, par une étude plus spécifique de l'influence du revêtement du sol sur la colonisation des troncs par les lichens saxicoles. D'autres inventaires sur des arbres d'alignement hors des jardins de Paris pourraient également être réalisés afin d'améliorer les connaissances sur la diversité lichénique corticole de Paris.

En zone urbanisée, la distribution spatiale des lichens corticoles est un axe d'étude multifactoriel. Plusieurs facteurs structurants sont pressentis: conditions écologiques microstationnelles, types de phorophytes présents, qualité de l'air, etc. Des études statistiques viendront étayer ces premiers résultats et feront l'objet de publications futures.

\section{Remerciements}

Cette étude a été financée par l'Institut d'Aménagement et d'Urbanisme de la région Île-de-France (IAU IdF) et par une Action transversale du Muséum (ATM)

Nous remercions chaleureusement toutes les personnes qui nous ont aidés dans ce travail, et plus particulièrement, Chantal Van Haluwyn et Claude Roux pour leurs conseils et leur aide dans la détermination des lichens (notamment T. populorum, C. arnoldii, M. albescens, M. crenulata, R. genarii et $X$. huculica); Nicolas Magain et son équipe (Université de Liège) pour le séquençage ADN de $E$. adsendens; Michel Bertrand pour l'identification de C. flavocitrina; et André Aptroot pour l'identification et la confirmation des espèces de Chèvreloup du genre Bacidia et Catillaria. Ces remerciements s'adressent également aux personnes qui nous ont accompagnés sur le terrain, Laure Turcati, Yannick Agnan, Sébastien Filoche et Rémy Poncet. Merci à Didier Jacquin, Gisèle Croq du Sénat pour nous avoir donné l'autorisation de faire un inventaire des lichens corticoles et des prélèvements pour l'herbier PC au jardin du Luxembourg. Merci à Chantal Van Haluwyn et Rémy Poncet, les rapporteurs, pour la relecture de cet article.

\section{RÉFÉRENCES}

Belair D. 1977. - Contribution à l'étude de la distribution des lichens épiphytes dans L'Île-de-France: application au problème de la pollution atmosphérique. Mémoire de Thèse, Université de Lille II, 394 p. 
Bokhorst S., Convey P., Huiskes A. \& Aerts R. 2016. Usnea antarctica, an important Antarctic lichen, is vulnerable to aspects of regional environmental change. Polar Biology 39 (3): 511-521. https://doi.org/10.1007/s00300-015-1803-z

Bouly DE LeSDAIN M. 1905. - Lichens des environs de Versailles. Bulletin de la Société botanique de France 52 (8): 602 628. https://doi.org/10.1080/00378941.1905.10829200

Bouly DE LeSDAIN M. 1907. — Lichens des environs de Versailles. Bulletin de la Société botanique de France 54 (8): 680698. https://doi.org/10.1080/00378941.1907.10833382

Bouly De LesDain M. 1908. - Lichens des environs de Versailles (2e supplément). Bulletin de la Société botanique de France 55 (9): 687-694. https://doi.org/10.1080/0037894 1.1908 .10832049

Bouly DE LESDAIN M. 1912. — Lichens des environs de Versailles (3e supplément). Bulletin de la Société botanique de France 59 (1): 11-18. https://doi.org/10.1080/00378941.1912.10832375

Bouly DE LesDain M. 1921. — Lichens des environs de Versailles (Quatrième et dernier supplément). Bulletin de la Société botanique de France 68 (1): 16-24. https://doi.org/10.1080/ 00378941.1921 .10836736

Bouly De LeSDAIN M. 1948. - Ecologie (phanérogames, mousses et lichens) de quelques sites de Paris. Le Chevalier, Paris, $87 \mathrm{p}$.

Cáceres M. E. D., Aptroot A. \& LǗcking R. 2017. — Lichen fungi in the Atlantic rain forest of Northeast Brazil: the relationship of species richness with habitat diversity and conservation status. Brazilian Journal of Botany 40: 145-156. https://doi.org/10.1007/s40415-016-0323-6

Chevallier F. F. 1836. - Flore générale des environs de Paris, selon la méthode naturelle. Imprimerie Baudouin, Paris, $617 \mathrm{p}$.

Clauzade G. \& Roux C. 1985. - Likenoj de Okcidenta Eŭropo: ilustrita determinlibro. Bulletin de la Société botanique du Centre-Ouest 7: 1-893.

Coste C. 1991. - Initiation à l'étude des lichens. Bulletin de l'Association française de Lichénologie 16 (1): 3-11.

Daniels F. J. A., Bültmann H., Lünterbusch C. \& Wilhelm M. 2000. - Vegetation zones and biodiversity of the NorthAmerican arctic. Berichte der Reinhold - Tüxen - Gesellschaft 12: $131-151$.

Deruelle S. 1983. - Écologie des lichens du Bassin parisien: impact de la pollution atmosphérique (engrais, SO2, $\mathrm{Pb}$ ) et relations avec les facteurs climatiques. Thèse de doctorat d'état, Université Pierre et Marie Curie, Paris, 360 p.

Doignon P. 1947. — Lichens inédits du Massif de Fontainebleau. Bulletin de la Société botanique de France 94 (7-8): 222-223. https://doi.org/10.1080/00378941.1947.10834617

Fredon F., Letrouit-Galinou M.-A. \& Avnaim M. 1993. Recolonisation par les lichens des arbres de Paris. Bulletin d'Information de l'Association française de Lichénologie 2: 43-60

GIORDANI P. 2007. - Is the diversity of epiphytic lichens a reliable indicator of air pollution? A case study from Italy. Environmental Pollution 146 (2): 317-323. https://doi.org/10.1016/j. envpol.2006.03.030

Goldhaber F. \& RAMdane S. 2018. - Le Muséum national d'Histoire naturelle a accueilli près de 3 millions de visiteurs en 2017. [Communiqué de presse]

Ishiomin F. \& Letrouit-Galinou M.-A. 1997. — Les lichens corticoles de Paris et la pollution. Évolution des données 19911993-1995. Rapport de maîtrise B.P.E: L'Homme et la Biosphère. Institut d'écologie, Paris, $32 \mathrm{p}$.

Kristinsson H., ZhurbenKo M. \& Hansen E. S. 2010. - Panarctic Checklist of Lichens and Lichenicolous Fungi. CAFF Technical Report No. 20. CAFF International Secretariat, Akureyri, 120 p.

LeBlanc S. F. \& Sloover J. D. 1970. - Relation between industrialization and the distribution and growth of epiphytic lichens and mosses in Montreal. Canadian Journal of Botany 48 (8): 1485-1496. https://doi.org/10.1139/b70-224

Lebreton E., Rivart S., Leblond S. \& Meyer C. 2018. -
Suivi spatio-temporel des communautés lichéniques à Paris en lien avec les pressions environnementales. Rapport de master B2E, PatriNat (OFB, CNRS, MNHN), Paris, 46 p.

Llewellyn T., Gaya E. \& Murrell D. J. 2020. - Are urban communities in successional stasis? A case study on epiphytic lichen communities. Diversity 12 (9): 330. https:// doi.org/10.3390/d12090330

LÜCKING R., Hodkinson B. P. \& LeavitT S. D. 2016. - The 2016 classification of lichenized fungi in the Ascomycota and Basidiomycota - Approaching one thousand genera. The Bryologist 119 (4): 361-416. https://doi.org/10.1639/00072745-119.4.361

McMullin R. T. \& Anderson F. 2014. - Common Lichens of Northeastern North America. Memoirs of the New York Botanical Garden 112: 1-180.

Mérat F. V. 1812. - Nouvelle flore des environs de Paris. Imprimerie de Crapelet, Paris, 420 p.

NF EN 16413 2014. - Air ambiant-Biosurveillance à l'aide de lichens - Évaluation de la diversité de lichens épiphytes. Agence française de normalisation, Paris, 32 p.

Nylander W. 1866. - Les lichens du jardin du Luxembourg. Bulletin de la Société botanique de France 13 (7): 364-371. https://doi.org/10.1080/00378941.1866.10827433

Nylander W. 1896. — Les lichens des environs de Paris. Imprimerie Paul Schmidt., Paris, 142 p.

Particitae 2019. - Livret de Lichen Go! Évaluez la qualité de l'air en observant les lichens. Particitae, Paris, 20 p.

Pinho P., Augusto S., Branquinho C., Bio A., Pereira M. J., SOARES A. \& CATARINO F. 2004. - Mapping lichen diversity as a first step for air quality assessment. Journal of Atmospheric Chemistry 49: 377-389. https://doi.org/10.1007/s10874004-1253-4

Purvis W. 2000. - Lichens. The Natural History Museum, London, $112 \mathrm{p}$.

RICHARDSON D. H. S. 1975. - The Vanishing Lichens: Their History, Biology and Importance. David \& Charles Publishers, London, Vancouver, $240 \mathrm{p}$.

Richardson D. H. S. \& CAmERon R. P. 2004. - Cyanolichens: their response to pollution and possible management strategies for their conservation in Northeastern North America. North eastern Naturalist 11 (1): 1-22. https://doi.org/10.1656/10926194(2004)011[0001:CTRTPA]2.0.CO;2

Roux C. (éd.) 2017. - Catalogue des lichens et champignons lichénicoles de France métropolitaine. $2^{e}$ édition revue et augmentée. Association française de lichénologie (A.F.L.), Fontainebleau, $1581 \mathrm{p}$

Roux C. (éd.) 2020. - Catalogue des lichens et champignons lichénicoles de France métropolitaine. $3 e$ édition revue et augmentée. Association française de lichénologie (A.F.L.), Fontainebleau, $1769 \mathrm{p}$.

Ryan B. D., Lumbsch H. T., Messuti M. I., Printzen C., Oeliwa I., Nash T. H. \& Bungartz F. 2004. - Lichen Flora of the Greater Sonoran Desert Region. Lichens Unlimited, Arizona State University, $742 \mathrm{p}$.

Sipman H. J. \& Aptroot A. 2001. - Where are the missing lichens? Mycological Research 105 (12): 1433-1439. https:// doi.org/10.1017/S0953756201004932

SLOOF J. E. 1993. - Environmental Lichenology: Biomonitoring Trace-Element Air Pollution. Interfacultair Reactor Insituut, Delft University of Technology, $189 \mathrm{p}$.

Smith C. W., Aptroot A., Coppins B. J., Fletcher A., GilBert O. L., James P. W. \& Wolseley P. A. 2009. - The Lichens of Great Britain and Ireland. British Lichen Society. The Natural History Museum, London, 1046 p.

Tournefort J. P. D. 1698. - Histoire des Plantes qui naissent aux environs de Paris, avec leur usage dans la Médecine. Imprimerie royale, Paris, $534 \mathrm{p}$.

UICN FRANCE 2013. - Panorama des services écologiques four- 
nis par les milieux naturels en France. Vol. 2.3 Les écosystèmes urbains. UICN France, Paris, 20 p.

VAn HaluWyn C. \& ASTA J. 2013. - Guide des lichens de France.
Lichens des arbres. Belin, Saint-Just-la-Pendue, $231 \mathrm{p}$.

Wirth V., Hauck M., Schultz M., \& De BruYN U. 2013. -

Die Flechten Deutschlands: tome $1 \& 2$. Ulmer, Stuttgart, 1244 p.

Soumis le 11 octobre 2020; accepté le 8 février 2021; publié le 3 novembre 2021. 